\title{
An Effectiveness Study on the Class Lecture in An ePUB3 eBook-based Flip Blended Learning Model
}

\author{
Tina Pingting Tsai ${ }^{1}$, Jyhjong Lin ${ }^{2}$, Lendy Chaoyu $\operatorname{Lin}^{3}$, Yihsiu Chen ${ }^{4}$, and Jingting Liu ${ }^{5}$ \\ ${ }^{1}$ General Education Center, Hsuan Chuang University, Taiwan \\ 2,3,4,5 Department of Information Management, Ming Chuan University, Taiwan \\ 1'tinatsai85@hcu.edu.tw, ${ }^{2}$ jlin@mail.mcu.edu.tw, ${ }^{3}$ lendy.lin@gmail.com, ${ }^{4}$ mi04crazy@gmail.com, ${ }^{5}$ sgn95420@gmail.com
}

\begin{abstract}
In e-Learning, the flip blended learning model is commonly used to provide students with learning contents inside/outside classrooms. It encourages students to preview learning contents at their own pace outside classrooms before the class lecture. Thereafter, student-centric actions are taken inside classrooms in the lecture with instructions/helps from the teacher. An important issue about its success is the effectiveness review of a class lecture to explore its possible weaknesses. In addition, for an ePUB3 eBook-based flip blended learning model, ePUB3 eBooks are used in the model for students to access learning contents during the class lecture. The embedded ePUB3 track and test functions in these eBooks can be applied for tracking students' in-lecture accesses on these eBooks and examining their post-lecture learning outcomes. As such, the teacher can review the effectiveness of the class lecture to explore its possible weaknesses. In this paper, we explore such a study on the effectiveness of class lectures via ePUB3 track and test functions in an ePUB3 eBook-based flip blended learning model. For illustration, this study is applied to an academic 'Script Writing' course and respective discussions are presented for showing its usefulness.
\end{abstract}

Key words: flip blended learning, class lecture, effectiveness, ePUB3 eBook

\section{Introduction}

In e-Learning, many efforts have been made to build web-/ cloud-based learning management systems (LMS) [1] such as SCORM [2] that provide controllable processes of learning activities with desired curricular contents delivered to desired learners. In general, these approaches support well the provision of a controlled process of learning activities. They however do not address the delivery ways of desired contents in the controlled process; in addition, they have no discussions about the implementation of such designated delivery ways by using selected publication formats and the verification of these constructed delivery functions by corresponding contents readers.

For this need, we presented earlier in [3] some discussions about the delivery ways of desired contents, the implementation of these ways by ePUB3 eBooks [4]. Afterwards, by using these functions in blended learning $[5,6]$, we proposed in a recent study [7] a flip blended learning approach for designing and lecturing academic courses with eBook-based ePUB3 functions used in learning activities for delivering desired curricular contents.

However, according to our experiences on using the flip blended learning model, an issue about its success was not well discussed: the effectiveness review of a class lecture to capture its possible weaknesses. In our view, this issue is important because it can help the teacher to improve his/her later class lectures based on the captured lecture weaknesses. Therefore, we explore in this paper this issue by discussing the possible effectiveness conditions of a class lecture such that the teacher can capture its possible weaknesses. In particular, since ePUB3 eBooks are used in the flip blended learning model where ePUB3 track and test functions are embedded for tracking students' in-lecture accesses on these eBooks and examining their post-lecture learning outcomes, our discussions will therefore focus on the uses of these ePUB3 track and test functions and how they are addressed to derive the possible effectiveness conditions (by checking these accesses and their relationships with the test outcomes).

\section{Study on Lecture Effectiveness}

\section{A. Effectiveness Conditions of A Class Lecture}

Since the effectiveness of students' learning in the class lecture can be derived from exploring the relationships between students' in-lecture accesses on eBooks and their post-lecture test results, it therefore may have the following four possible conditions:

1. The lecture is actively effective with respect to a specific part of earning contents in these eBooks in that they have well been accessed and learned. In general, this condition is derived from (1) students take sufficient accesses on this part, e.g., viewing textual pages or using embedded modalities over required thresholds of such determinants as time points and number of times; and (2) students possess qualified test results, e.g., over the required 80 average score points at the test, with regard to this part. It should be noted that for ensuring the sufficiency of students' accesses, the teacher needs to set up required thresholds for those determinants applicable to these accesses. For example, viewing a textual page may be said sufficient if the ratio of all students' viewing in the lecture (time points) is $\geq 75 \%$. In addition, using an embedded modality may be said sufficient if the ratio of all students' using at least once (number of times) is $\geq 60 \%$.

2. The lecture is passively effective with respect to a specific part of learning contents in these eBooks in that they have not yet been well accessed but already been well learned. In general, this condition is derived from (1) students take insufficient accesses (i.e., not reaching required thresholds of applicable determinants) on these contents; and (2) students still possess qualified test results with regard to these contents. 
3. The lecture is actively ineffective with respect to a specific part of learning contents in that they have been well accessed but not yet been well learned. In general, this condition is derived from (1) students take sufficient accesses (i.e., reaching required thresholds of applicable determinants) on these contents; and (2) students possess unqualified test results with regard to these contents.

4. The lecture is passively ineffective with respect to a specific part of learning contents in that they have not yet well been accessed and learned. In general, this condition is derived from (1) students take insufficient accesses (i.e., not reaching required thresholds of applicable determinants) on these contents; and (2) students possess unqualified test results with regard to these contents.

\section{B. Effectiveness Comprehension of The Class Lecture}

With the possible effectiveness conditions of the class lecture, it is time to explore the causes of these conditions and then attribute these causes to the possible weaknesses in learning contents or lecture actions. As one may conceive, these weaknesses may help the teacher to make amendments on these contents or actions for alleviating these weaknesses in his/her later lectures.

1. For an actively effective lecture with respect to a specific part of learning contents, since these contents have well been accessed and learned, the purpose of the lecture has been achieved after students take sufficient accesses. Therefore, this implies that (1) these contents are suitable for the students to learn; and (2) actions for learning these contents are good for students to learn without explicit difficulties. In this condition, these contents may be considered as adequate for students to learn and hence can be reused in later lectures. Further, for these actions good for students' learning, the teacher may also reuse them in later lectures.

2. For a passively effective lecture with respect to a specific part of learning contents, since these contents have not yet been well accessed but already been well learned, the purpose of the lecture has been achieved although students take insufficient accesses. Therefore, this implies that (1) these contents are not suitable (e.g., too simple) for students such that they do possess the knowledge in these contents with no needs of sufficient accesses; and (2) actions for learning these contents are good for students without explicit difficulties. In this condition, the teacher may consider to increase the difficult degree of these contents for making them adequate for students to learn in later lectures. Further, for these actions good for students' learning, the teacher may also reuse them in later lectures.

3. For an actively ineffective lecture with respect to a specific part of learning contents, since these contents have been well accessed but not yet been well learned, the purpose of the lecture has not been achieved although students take sufficient accesses. Therefore, this implies that (1) these contents are not suitable (e.g., too difficult) for students such that they do not possess the knowledge in these contents even if they take sufficient accesses; and (2) actions for learning these contents are not good enough for students to learn well without explicit difficulties. In this condition, the teacher may consider to decrease the difficult degree of these contents for making them adequate for students to learn in later lectures. Further, for these actions not good enough for students' learning, the teacher may need to adapt them or adopt other ones in later lectures.

4. For a passively ineffective lecture with respect to a specific part of learning contents, since these contents have not yet well been accessed and learned, the purpose of the lecture has not been achieved under students' taking insufficient accesses. Therefore, this implies that (1) it is not easy to conclude whether these contents are suitable or not for students due to insufficient accesses; and (2) actions for learning these contents are not good enough for students to learn well without explicit difficulties. In this condition, the teacher may need to issue some effective ways for encouraging students to take sufficient accesses in later lectures. Further, for these actions not good enough for students' learning, the teacher may need to adapt them or adopt other ones in later lectures.

\section{Application of The Study}

In this section, we apply our study on an academic 'Script Writing' course at Hsuan Chuang University in Taiwan.

\section{A. Design and Construction of ePUB3 eBook}

As shown in Table 1, the 'Script Writing' course has six learning activities where the supportive ePUB3 functions are also described. Afterwards, based on these course designs, the ePUB3 eBooks used in the course can be constructed by View Porter [8] which has been well recognized as a powerful tool for constructing high quality ePUB3 eBooks.

\section{B. Design and Construction of ePUB3 eBook}

After designing the 'Script Writing' course and constructing the ePUB3 eBooks used in the course, a class of this course is then taken as scheduled at the Spring semester in 2017 where 62 students have enrolled and 4 ePUB3 eBooks have been used in its six learning activities - (1) a Lecture eBook for the preview, class lecturing, and exercises, (2) a Pre-Lecture Test eBook for the pre-lecture test, (3) a Post-Lecture Test eBook for the post-lecture test, and (4) a Questionnaire eBook for the questionnaire. Therefore, after the class lecture and postlecture test activities have been completed with enrolled students viewing the curricular contents and taking the postlecture test via Lecture \& Post-Lecture Test eBooks, the actual conditions about the lecture effectiveness can then be identified for exploring the possible weaknesses in the lecture.

For this, students' in-lecture accesses on the Lecture eBook (e.g., viewing its textual pages / using its embedded modalities) are tracked by such determinants as time points and number of times. Further, for ensuring the sufficiency of these accesses, respective thresholds are set up for those determinants applicable to these accesses. As shown in Table 2, students' accesses are tracked by two determinants: $\mathrm{T}$ denotes the time point and $\mathrm{N}$ means the number of times. The sufficiency of these accesses on a specific part of the Lecture eBook (i.e., viewing two-consecutive-pages of its textual pages or using one of its embedded modalities) is determined by the ratio of all students' accesses reaching the thresholds of these determinants. 
Table 1: design of the 'Script Writing' course (learning activities and supportive ePUB3 functions)

\begin{tabular}{|c|c|c|c|c|c|c|c|}
\hline Course Name & \multicolumn{7}{|l|}{ Script Writing } \\
\hline \multirow{2}{*}{$\begin{array}{c}\text { Flip } \\
\text { Learning } \\
\text { Model }\end{array}$} & before class & begin of class & \multicolumn{2}{|c|}{ lecture in class } & \multicolumn{2}{|c|}{ end of class } & after class \\
\hline & $\begin{array}{c}\text { outside-class } \\
\text { room } \\
\text { preview }\end{array}$ & $\begin{array}{c}\text { inside-classroom } \\
\text { pre-lecture test }\end{array}$ & $\begin{array}{l}\text { inside-classr } \\
\text { lecturing anc }\end{array}$ & $\begin{array}{l}\text { m class } \\
\text { xercise }\end{array}$ & \multicolumn{2}{|c|}{$\begin{array}{l}\text { inside-classroom } \\
\text { post-lecture test }\end{array}$} & \begin{tabular}{|c|} 
inside- \\
classroom \\
questionnaire
\end{tabular} \\
\hline \multirow{2}{*}{$\begin{array}{l}\text { Learning } \\
\text { Activities }\end{array}$} & \multicolumn{7}{|c|}{ ePUB3 functions } \\
\hline & videos & links & exercises & \multicolumn{2}{|c|}{ tests } & \multicolumn{2}{|c|}{ questionnaires } \\
\hline preview & $V$ & $V$ & $V$ & & & & \\
\hline pre-lecture test & $V$ & & & & 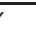 & & \\
\hline lecturing & $V$ & $V$ & & & & & \\
\hline exercises & & & $V$ & & & & \\
\hline post-lecture test & & & & & & & \\
\hline questionnaire & & & & & & & $V$ \\
\hline
\end{tabular}

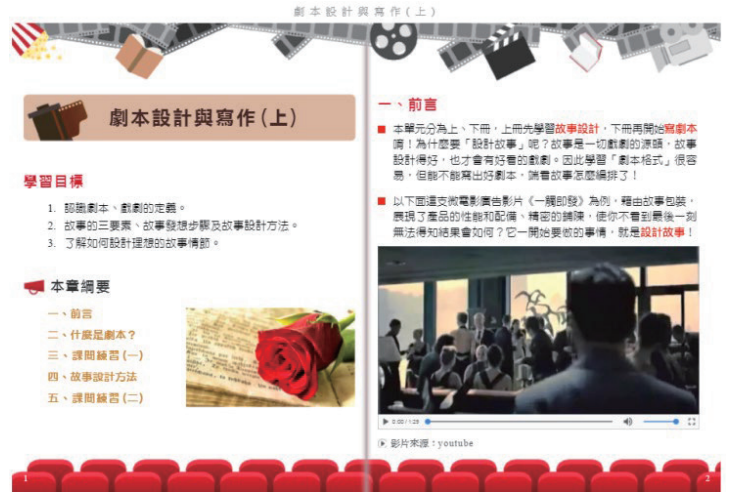

Figure 1: part of the Lecture ePUB3 eBook

Table 2: students' accesses on the Lecture eBook

\begin{tabular}{||c|c|c|c|c||}
\hline \multicolumn{5}{||c||}{ Lecture ePUB3 eBook } \\
\hline page & $\begin{array}{c}\text { page } \\
\text { views }\end{array}$ & videos & links & exercises \\
\hline $1-2$ & $\mathrm{~T}: 86 \%$ & $\mathrm{~N}: 54 \%$ & & \\
\hline $3-4$ & $\mathrm{~T}: 89 \%$ & & $\mathrm{~N}: 75 \%$ & $\mathrm{~N}: 78 \%$ \\
\hline $5-6$ & $\mathrm{~T}: 94 \%$ & & & \\
\hline $7-8$ & $\mathrm{~T}: 94 \%$ & $\mathrm{~N}: 64 \%$ & & \\
\hline $9-10$ & $\mathrm{~T}: 94 \%$ & & & \\
\hline $11-12$ & $\mathrm{~T}: 86 \%$ & $\mathrm{~N}: 53 \%$ & & \multirow{2}{*}{$\mathrm{N}: 75 \%$} \\
\hline 13 & $\mathrm{~T}: 78 \%$ & & & \\
\hline
\end{tabular}

Table 3: outcomes of students' post-lecture test

\begin{tabular}{|c|c|c|c|c|c|c|c|}
\hline \multicolumn{8}{|c|}{ post-lecture test for Lecture ePUB3 eBook } \\
\hline \multirow[t]{2}{*}{ scores } & views & \multicolumn{3}{|c|}{ videos } & \multirow{2}{*}{$\begin{array}{l}\text { links } \\
\text { p.3-4 }\end{array}$} & \multicolumn{2}{|c|}{ exercises } \\
\hline & all pages & p. $1-2$ & p.7-8 & p.11-12 & & p.3-4 & p.11-13 \\
\hline highest & 100 & 100 & 100 & 100 & 100 & 100 & 100 \\
\hline average & 82 & 76 & 80 & 85 & 86 & 86 & 80 \\
\hline lowest & 35 & 40 & 40 & 40 & 20 & 20 & 40 \\
\hline
\end{tabular}

For example, reading textual pages 1-2 is said sufficient if the ratio of all students' reading in the lecture ( $\mathrm{T}-$ in the lecture) is higher than $75 \%$. In addition, watching an embedded video on pages 1-2 is said sufficient if the ratio of all students' watching at least once ( $\mathrm{N}-$ at least once) is higher than $60 \%$. As results in Table 2, all readings of textual pages are sufficient in that they reach the threshold of $75 \%$ (i.e., all are $78 \%-94 \%$ ). Further, watching an embedded video on pages 1-2 is not sufficient due to its ratio $54 \%$ lower than the required $60 \%$ threshold. However, it is observed that watching an embedded video on pages $7-8$ is sufficient due to its ratio $64 \%$ higher than the $60 \%$ threshold.

Further, for deriving the achieved effectiveness conditions of this lecture, the outcomes of the post-lecture test are collected as shown in Table 3 where students possess (1) a qualified 82 test result, i.e., over the required 80 average score points at the test, with regard to their reading all textual pages for learning how to design stories and convert them into scripts; (2) unqualified $76 /$ qualified $80 /$ qualified 85 test results with regard to their watching the embedded videos on pages 1-2/ 7-8/11-12 for learning more generic concepts about story design and script writing; (3) a qualified 86 test result with regard to their accessing the embedded referential links on pages 3-4 for referencing additional materials about story design and script writing; and (4) qualified 86 / 80 test results with regard to their taking the embedded exercises on pages 3-4 / 11-13 for reviewing the necessary knowledge about story design and script writing.

In summary, with the above tracked students' in-lecture accesses in Table 2 and their post-lecture test results in Table 3 , the achieved effectiveness conditions of this lecture can then be identified as follows.

1. The lecture is actively effective with respect to reading all textual pages in that students have well read all textual pages and well learned the focuses of these pages on how to design stories and convert them into scripts. Thus, in this condition, these pages are adequate for students to learn and hence can be reused in later classes. Further, since reading these pages is good for students, the teacher can reuse it in later classes.

2. The lecture is passively ineffective with respect to watching the embedded video on pages 1-2 in that students have not yet well watched this video and also not yet well learned the focus of this video on more generic concepts about story design and script writing. Thus, in this condition, the teacher can consider to issue some effective ways for encouraging students to watch this video in later classes. Further, since watching this video is not good for students, the teacher can consider to adapt this action or adopt other ones in later classes.

3. The lecture is actively effective with respect to watching the embedded video on pages 7-8 in that students have well watched this video and also well learned the focus of this 
video on more generic concepts about story design and script writing. Since this condition is the same as that in the above reading all textual pages, its implications are also the same as those in that condition.

4. The lecture is passively effective with respect to watching the embedded video on pages 11-12 in that students have not yet well watched this video but well learned the focus of this video on more generic concepts about story design and script writing. Thus, in this condition, the teacher can consider to increase the difficult degree of this video for making them adequate for students to learn in later classes. Further, since watching this video is good for students, the teacher can reuse it in later classes.

5. The lecture is actively effective with respect to accessing the embedded referential links on pages 3-4 in that students have well accessed these links and well learned the focuses of these links on the additional knowledge about story design and script writing. Since this condition is the same as that in the above reading all textual pages, its implications are also the same as that condition.

6. The lecture is actively effective with respect to taking the embedded exercises on pages 3-4 and 11-13 in that students have well taken these exercises and well learned the focuses of these exercises on reviewing the necessary knowledge about story design and script writing. Since this condition is the same as that in the above reading all textual pages, its implications are also the same as that condition.

\section{Conclusions}

In this paper, we discuss an important issue about the success of using the ePUB3 eBook-based flip blended learning model: the effectiveness review of a class lecture to explore its possible weaknesses. In particular, our discussions focus on the uses of ePUB3 track and test functions and how they are addressed to capture the lecture effectiveness. Therefore, in terms of the relationships between in-lecture accesses and post-lecture test, the lecture effectiveness can be identified in four possible conditions: (1) actively effective; (2) actively ineffective; (3) passively effective; and (4) passively ineffective. Afterwards, the causes of these conditions can then be explored to comprehend the possible weaknesses in learning contents or lecture actions. Then, based on these weaknesses, the teacher can make amendments on these contents or actions for alleviating these weaknesses in later classes.

For illustration, our discussions are applied to an academic 'Script Writing' course at Hsuan Chuang University in Taiwan. A class of this course is taken as scheduled at the Spring semester in 2017 where 62 students have enrolled and two Lecture and Post-Lecture Test eBooks are respectively used for its lecture and test activities. After then, the actual conditions about this class's lecture effectiveness have been identified and as a result their causes for capturing the possible weaknesses in contents or actions have also been explored.

\section{References}

[1] Stone, D. and Zheng, G., 2014, Learning Management Systems in a Changing Environment, Handbook of Research on Education and Technology in a Changing
Society, chapter 56, IGI Global.

[2] SCORM 2004, 4th Edition, https://www.adlnet.gov/adlresearch/scorm/scorm-2004-4th-edition/.

[3] Lin, L., et al., 2017, Some Useful ePUB3-based Contents Delivery Functions, Proc. of 5th International Conference on Information and Education Technology, pp. 49-52.

[4] ePUB3 Overview, 2011, http://www.idpf.org/epub/30/spec/.

[5] Bonk, C. and Graham, C., 2006, The Handbook of Blended Learning, Wiley.

[6] Bonk, C. and Graham, C., 2005, Handbook of Blended Learning: Global Perspectives, Local Designs, Pfeiffer Publishing.

[7] Tsai, T., et al., 2018, A Flip Blended Learning Approach for ePUB3 eBook-based Course Design and Implementation, Eurasia Journal of Mathematics, Science and Technology Education, 14(1), pp. 123-144.

[8] ViewPorter, 2017, ePUB3 Editor, Mercury v2.3, http:// viewporter.com. 